\title{
Inotersen preserves or improves quality of life in hereditary transthyretin amyloidosis
}

\author{
Teresa Coelho ${ }^{1} \cdot$ Aaron Yarlas $^{2}(1) \cdot$ Marcia Waddington-Cruz ${ }^{3} \cdot$ Michelle K. White $^{2} \cdot$ Asia Sikora Kessler $^{2}$. \\ Andrew Lovley ${ }^{2} \cdot$ Michael Pollock ${ }^{4}$. Spencer Guthrie ${ }^{5}$ Elizabeth J. Ackermann ${ }^{6}$. Steven G. Hughes ${ }^{7}$. Chafic Karam ${ }^{8}$. \\ Sami Khella ${ }^{9} \cdot$ Morie Gertz ${ }^{10}$. Giampaolo Merlini ${ }^{11}$ - Laura Obici ${ }^{11}$. Hartmut H. Schmidt ${ }^{12}$. Michael Polydefkis ${ }^{13}$. \\ P. James B. Dyck ${ }^{14}$. Thomas H. Brannagan III ${ }^{15}$. Isabel Conceição ${ }^{16} \cdot$ Merrill D. Benson $^{17}$. John L. Berk ${ }^{18}$
}

Received: 23 October 2019 / Revised: 6 December 2019 / Accepted: 9 December 2019 / Published online: 18 December 2019

(c) The Author(s) 2019

\begin{abstract}
Objective To examine the impact on quality of life (QOL) of patients with hATTR amyloidosis with polyneuropathy treated with inotersen (Tegsedi ${ }^{\mathrm{TM}}$ ) versus placebo.

Methods Data were from the NEURO-TTR trial (ClinicalTrials.gov Identifier: NCT01737398), a phase 3, multinational, randomized, double-blind, placebo-controlled study of inotersen in patients with hATTR amyloidosis with polyneuropathy. At baseline and week 66, QOL measures-the Norfolk-QOL-Diabetic Neuropathy (DN) questionnaire and SF-36v2 ${ }^{\circledR}$ Health Survey (SF-36v2) - were assessed. Treatment differences in mean changes in QOL from baseline to week 66 were tested using mixed-effect models with repeated measures. Responder analyses compared the percentages of patients whose QOL meaningfully improved or worsened from baseline to week 66 in inotersen and placebo arms. Descriptive analysis of item responses examined treatment differences in specific activities and functions at week 66.

Results Statistically significant mean differences between treatment arms were observed for three of five Norfolk-QOL-DN domains and five of eight SF-36v2 domains, with better outcomes for inotersen than placebo in physical functioning, activities of daily living, neuropathic symptoms, pain, role limitations due to health problems, and social functioning. A larger percentage of patients in the inotersen arm than the placebo arm showed preservation or improvement in Norfolk-QOL-DN and SF-36v2 scores from baseline to week 66. Responses at week 66 showed more substantial problems with daily activities and functioning for patients in the placebo arm than in the inotersen arm.

Conclusion Patients with hATTR amyloidosis with polyneuropathy treated with inotersen showed preserved or improved QOL at 66 weeks compared to those who received placebo.
\end{abstract}

Keywords Transthyretin amyloidosis · Quality of life $\cdot$ Polyneuropathy $\cdot$ Physical function $\cdot$ Rare disease

\section{Introduction}

Hereditary transthyretin (hATTR) amyloidosis is a rare, systemic, progressive, debilitating, and fatal disease characterized by mutations in the gene encoding the transthyretin (TTR) protein [1]. More than 130 reported TTR gene mutations promote misfolding of TTR proteins, which aggregate and deposit as insoluble amyloid deposits in tissues, inducing organ damage [2]. Worldwide prevalence estimates indicate that approximately 50,000 people have been

Aaron Yarlas

ayarlas@qualitymetric.com

Extended author information available on the last page of the article diagnosed with hATTR amyloidosis, although it is thought to be significantly underdiagnosed [3]. Patients with hATTR amyloidosis typically suffer peripheral and autonomic nerve fiber injury, resulting in a length-dependent sensorimotor peripheral neuropathy, manifesting as pain, numbness, and weakness with eventual loss of ambulation. Others experience cardiomyopathy, characterized by conduction disease, thickening and stiffening of the ventricles, and eventual heart failure [1, 4-6]. The majority of patients, however, exhibit a mixed phenotype including nerve- and heart-related manifestations [7].

Patients with hATTR amyloidosis experience severely compromised quality of life (QOL) [8-11] that worsens with disease progression [12]. Furthermore, patients with 
hATTR amyloidosis with polyneuropathy have significantly impaired physical functioning and well-being $[9,10]$. Neuropathy-related QOL for patients with hATTR amyloidosis with polyneuropathy is comparable to patients with severe diabetic neuropathy that resulted in ulceration, gangrene, or amputations [11].

The NEURO-TTR trial was a phase 3 clinical trial that examined the safety and efficacy of inotersen (Tegsedi ${ }^{\mathrm{TM}}$ ) in patients with hATTR amyloidosis with polyneuropathy [13]. About two thirds of patients also had cardiomyopathy. The current research examines the impact of 66 weeks of inotersen treatment on patients' QOL recorded in the NEUROTTR trial.

\section{Methods}

\section{Sample and study characteristics}

The NEURO-TTR trial (ClinicalTrials.gov Identifier: NCT01737398) was a phase 3, multinational, randomized, double-blind, placebo-controlled study of inotersen in patients with hATTR amyloidosis with polyneuropathy. Patients were randomized to receive $300 \mathrm{mg}$ inotersen or placebo in a 2:1 ratio within each of three strata: presence/ absence of previous treatment with tafamidis and/or diflunisal; Stage 1 (ambulatory without assistance) or Stage 2 (ambulatory with assistance) neurologic disease severity, according to Coutinho et al.'s staging [14]; and Val30Met mutation or non-Val30Met mutation. Study drug was administered subcutaneously on three alternating days during the first week and once per week for the following 65 weeks.

Patients were eligible for the study if they were at least 18 years old, had Stage 1 or Stage 2 disease severity, had a neuropathy impairment score (NIS) between 10 and 130 (inclusive) at screening, had a documented TTR variant by genotyping; and had documented amyloid deposits by biopsy. Exclusion criteria included previous or anticipated liver transplant within 1 year of screening, a Karnofsky performance status $\leq 50$, a New York Heart Association (NYHA) functional classification $\geq 3$, or presence of type 1 or 2 diabetes, human immunodeficiency virus (HIV), hepatitis B, or hepatitis C.

Primary endpoints of the trial were changes from baseline to week 66 in the modified Neuropathy Impairment Score + 7 (mNIS + 7) [15-17] composite score and in the total score of the Norfolk QOL-Diabetic Neuropathy (DN) questionnaire [18], which has been validated in patients with ATTR accompanied by polyneuropathy [19]. A tertiary endpoint of the trial was change from baseline to week 66 in the physical component summary (PCS) score of the SF-36v2. Inotersen was shown to be significantly efficacious relative to placebo for each of these outcomes [13]. Safety outcomes for the NEURO-TTR trial have also been published [13].

The Norfolk QOL-DN and the SF-36v2 were administered to patients at baseline, week 35 , and week 66 (end of treatment). All analyses were based on the full analysis set, which was comprised of all randomized patients who received at least one injection of study drug and who had at least one post-baseline efficacy assessment for the mNIS + 7 score or Norfolk QOL-DN questionnaire total score.

\section{Outcomes}

Neuropathic-specific QOL was measured using the 35-item Norfolk QOL-DN [18]. It yields a total score based on all 35 items (score range from -4 to 136), and scores on five subscales capturing outcomes associated with damage to nerve fibers: activities of daily living (ADLs; five items, score range 0-20); autonomic neuropathy (three items, score range 0-12), large fiber neuropathy/physical functioning (15 items, score range -4-56); small fiber neuropathy (four items, score range 0-16); and symptoms (eight items, score range $0-32$ ). In all cases, higher scores indicate worse functioning.

Generic QOL was assessed using the SF-36v2 (with 4-week recall), a 36-item patient-reported outcome measure of functional health and well-being [20]. Responses to SF-36v2 items can be combined to compute scores for eight domains of QOL: physical functioning, role limitations due to physical health (role-physical), bodily pain, perception of general health, vitality, social functioning, role limitations due to emotional health (role-emotional), and mental health. Two summary scores-PCS, capturing global physical health, and the mental component summary (MCS) capturing global mental health-are calculated using weighted combinations of scores from all eight domains. All SF-36v2 domains and summary scores are expressed as $T$ scores using norm-based methods, standardized to a mean of 50 and a standard deviation of 10 in the general population. Higher SF-36v2 scores reflect better QOL. The SF-36v2 has been previously used in randomized-controlled trials comparing disease progression and QoL in patients with hATTR polyneuropathy [21].

Responder-level minimally important change (MIC) values have been established for SF-36v2 domain and summary scores using distribution-based methods. MIC threshold values, which have been defined as the smallest differences in scores that an individual patient would consider as beneficial and for which a clinician would recommend adjusting patients' care [22], can facilitate interpretation of whether a patient's change in health is clinically meaningful. 


\section{Statistical analysis}

All analyses described here were exploratory and post hoc.

Mixed-effects models for repeated measures (MMRM) were used to test for treatment arm differences in mean change-from-baseline scores for each domain of the Norfolk QOL-DN and SF-36v2 at week 66. Fixed class effects for all models included treatment arm, visit (week 35 and week 66), each of the three stratification factors-previous treatment with tafamidis and/or diflunisal (presence vs. absence), disease severity (Stage 1 vs. Stage 2), and mutation type (Val30Met mutation vs. non-Val30Met mutation) — and the treatment arm * visit interaction. Covariates included baseline score and the baseline score * visit interaction. Degrees of freedom were estimated using the Kenward-Rogers approach. An unstructured covariance model was used for within-subject residuals. Pairwise comparisons tested for treatment arm differences in leastsquares (LS) mean change scores at week 66.

Responder analysis examined the proportion of patients in each treatment arm who, at week 66, showed worse, same, or better scores from baseline for each SF-36v2 and Norfolk QOL-DN domain and summary measure. For SF-36v2 domain and summary scores, change status was determined based on established responder-level MICs. Patients whose SF-36v2 score at week 66 fell below their score at baseline by a magnitude of at least the MIC for that outcome were categorized as "worse," while those whose score at week 66 fell below their score at week baseline by less than the MIC for that outcome were categorized as "same/better." Patients whose SF-36v2 score at week 66 exceeded their score at baseline by a magnitude of at least the MIC for that outcome were categorized as "better."

For the Norfolk QOL-DN domains and total score, which do not have established responder-level MICs, change status was determined based on the distribution for each domain and total score. Specifically, status as "better," "same," or "worse" was determined using 0.5 of a standard deviation (SD) of the baseline score, as this magnitude has been identified as being indicative of an MIC in a score [23, 24]. Patients whose Norfolk QOL-DN score at week 66 met or exceeded their score at baseline by a magnitude of at least 0.5 of an SD for that outcome were categorized as "worse," while those who did not exceed their score at baseline by a magnitude of at least 0.5 of an SD for that outcome were categorized as "same/better." Patients whose Norfolk QOLDN score at week 66 fell below their score at baseline by a magnitude of at least 0.5 of an SD for that outcome were categorized as "better." For each SF-36v2 and Norfolk QOLDN score, the percentage of patients whose scores were categorized as same/better or better at week 66 than at baseline was compared across treatment groups using Fisher's exact tests.
To provide a more concrete interpretation of changes in QOL as a function of treatment, changes from baseline to week 66 in patients' responses to specific items of the Norfolk QOL-DN and SF-36v2 were assessed. The items included in the analyses ask how patients' physical and emotional health impacts patients' daily lives. For the Norfolk QOL-DN, items examine difficulty with ADLs and physical functioning; for the SF-36v2, items examine impairments in daily activities related to physical functioning, role-physical, bodily pain, and social functioning. Response choices for the selected items were dichotomized as indicating substantial and not substantial impairment. All examined Norfolk QOLDN items used response options of 'not a problem', 'very mild problem', 'mild problem', 'moderate problem', and 'severe problem', with the latter two options coded as indicating substantial problems. SF-36v2 physical functioning items use three response options, 'not limited at all', 'limited a little', and 'limited a lot'; only the last of these options was coded as indicating substantial impairment. Response choices for SF-36v2 role-physical items include 'all of the time', 'most of the time', 'some of the time', 'a little of the time', and 'none of the time'; substantial impairment was coded for the first two of these options. The SF-36v2 bodily pain item "How much did pain interfere with your normal work, including both work outside the home and housework?" has five possible responses: 'extremely', 'quite a bit', 'moderately', 'a little bit', and 'not at all'. Patients who indicated one of the first two responses were considered to have a substantial level of impairment, while those who selected one of the last three responses were considered to not have a substantial level of impairment. Analogous response choices were dichotomized similarly for the SF-36v2 social functioning item "During the past 4 weeks, to what extent has your physical health or emotional problems interfered with your normal social activities with family, friends, neighbors, or groups?" The percentages of patients whose itemlevel response indicated a substantial level of impairment at baseline and week 66 were calculated for patients in both treatment arms. Descriptive comparisons across treatment arms in the proportion of patients with responses indicating substantial impairment at baseline and at week 66 were examined to provide a real-world context for assessing the impact of inotersen on everyday functioning.

\section{Results}

Baseline characteristics of the analysis sample are presented in Table 1. Patients did not differ statistically by treatment arm for any of the listed characteristics. A majority of sample patients (62\%) had cardiomyopathy. Approximately twothirds of the sample (68\%) had Stage 1 neurologic disease severity; the remainder were Stage 2. 
Table 1 Baseline patient characteristics in the NEURO-TTR trial full analysis set $(n=165)$

\begin{tabular}{|c|c|c|c|}
\hline & Inotersen $(n=106)$ & Placebo $(n=59)$ & $\begin{array}{l}p \text { value (treatment } \\
\text { arm difference)* }\end{array}$ \\
\hline Age, mean (SD) & $59.6(12.4)$ & $59.4(14.1)$ & 0.937 \\
\hline Female, $N(\%)$ & $31(29.2)$ & $18(30.5)$ & 0.861 \\
\hline Mutation type, $N(\%)$ & & & 0.626 \\
\hline Val30Met mutation & $54(50.9)$ & $33(55.9)$ & \\
\hline Non-Val30Met mutation & $52(49.1)$ & $26(44.1)$ & \\
\hline Modified BMI, mean (SD) & $1025.3(222.7)$ & $1053.7(228.5)$ & 0.438 \\
\hline Presence of cardiomyopathy, $N(\%)$ & $70(66.0)$ & $32(54.2)$ & 0.181 \\
\hline Neuropathy stage, $N(\%)$ & & & 0.605 \\
\hline Stage 1 & $71(67.0)$ & $42(71.2)$ & \\
\hline Stage 2 & $35(33.0)$ & $17(28.8)$ & \\
\hline Duration of neuropathic symptoms in years, mean (SD) & $5.4(4.5)$ & $5.4(4.4)$ & 0.947 \\
\hline Prior treatment with tafamidis/diflunisal, $N(\%)$ & $62(58.5)$ & $35(59.3)$ & 1.000 \\
\hline mNIS + 7 total score, mean (SD) & $79.4(37.5)$ & $74.1(39.0)$ & 0.399 \\
\hline \multicolumn{4}{|l|}{ Norfolk QOL-DN, mean (SD) } \\
\hline Activities of daily living & $6.5(5.9)$ & $6.4(5.7)$ & 0.909 \\
\hline Autonomic neuropathy & $2.2(2.8)$ & $1.8(2.7)$ & 0.411 \\
\hline Large fiber/physical functioning & $24.1(15.4)$ & $24.4(13.7)$ & 0.891 \\
\hline Small fiber & $5.1(4.2)$ & $5.2(4.5)$ & 0.829 \\
\hline Symptoms & $10.6(6.1)$ & $10.7(6.5)$ & 0.974 \\
\hline Total score & $48.6(28.2)$ & $48.6(27.0)$ & 0.994 \\
\hline \multicolumn{4}{|l|}{ SF-36v2, mean (SD) } \\
\hline Physical functioning & $34.6(9.8)$ & $36.7(10.6)$ & 0.190 \\
\hline Role-physical & $37.2(10.7)$ & $38.2(10.2)$ & 0.575 \\
\hline Bodily pain & $43.5(9.8)$ & $42.6(10.4)$ & 0.582 \\
\hline General health & $40.8(8.8)$ & $43.1(9.1)$ & 0.112 \\
\hline Vitality & $45.9(10.0)$ & $46.5(11.1)$ & 0.720 \\
\hline Social functioning & $43.7(10.6)$ & $44.5(10.7)$ & 0.629 \\
\hline Role-emotional & $45.6(10.1)$ & $45.7(11.1)$ & 0.981 \\
\hline Mental health & $49.4(9.0)$ & $48.9(10.2)$ & 0.714 \\
\hline Physical component summary & 35.5 (8.9) & $37.2(9.9)$ & 0.267 \\
\hline Mental component summary & $51.1(9.2)$ & $50.6(10.7)$ & 0.774 \\
\hline
\end{tabular}

${ }^{*} p$ values (two-tailed) are based on independent-samples $t$ tests for continuous variables and Fisher's exact tests for categorical variables

Changes from baseline to week 66 in mean Norfolk QOLDN domain scores by treatment arm are presented in Fig. 1. Statistically significant differences in scores between treatment arms were observed for three domains: ADLs, large fiber/physical functioning, and symptoms, where stabilization or improvements in the inotersen arm were contrasted with worsening in the placebo arm, all $p \leq 0.01$.

Changes from baseline to week 66 in mean SF-36v2 domain scores by treatment arm are presented in Fig. 2 . Statistically significant differences between treatment arms in change-from-baseline scores at week 66 were observed for five domains: physical functioning, role-physical, bodily pain, social functioning, and role-emotional, where stabilization or improvements in the inotersen arm were again contrasted with worsening in the placebo arm, all $p<0.05$.

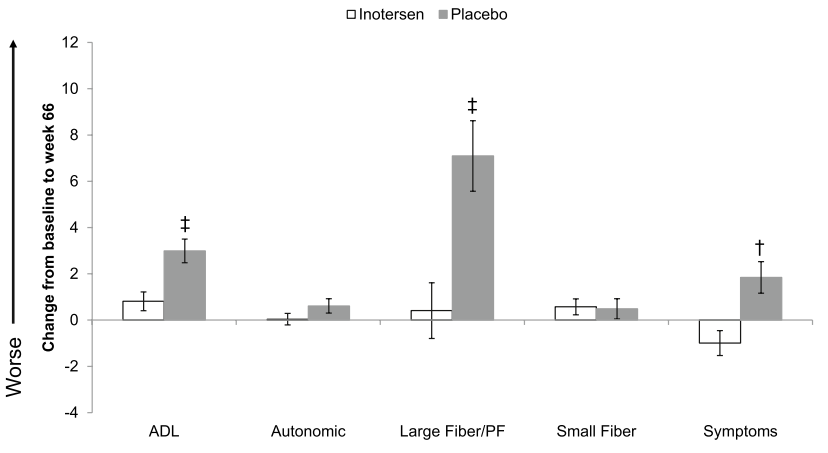

Fig. 1 Change in mean Norfolk QOL-DN Domain Scores from baseline to week 66 by treatment arm. $A D L$ activities of daily living, $P F$ physical functioning. Error bars represent standard errors $* p<0.05$, $p<0.01,{ }^{\ddagger} p<0.001$ 


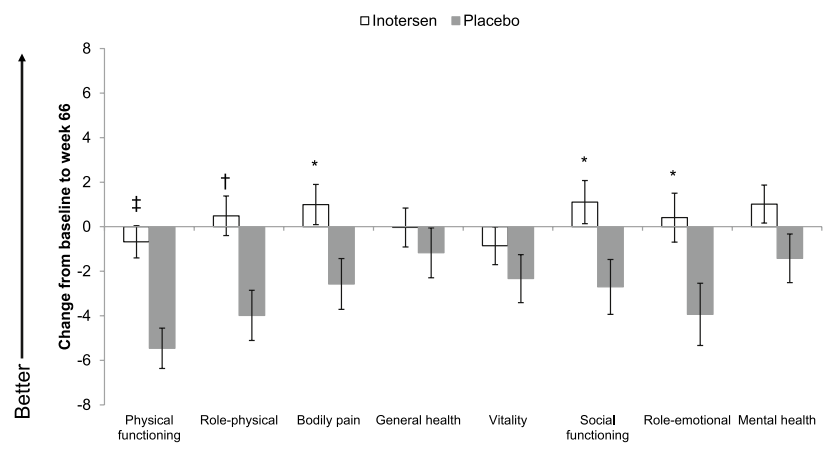

Fig. 2 Change in mean SF-36v2 Domain Scores from baseline to week 66 by treatment arm. Error bars represent standard errors ${ }^{*} p<0.05,{ }^{\dagger} p<0.01,{ }^{\ddagger} p<0.001$

Figure 3 presents the percentage of patients in each treatment arm whose Norfolk QOL-DN scores were the same or improved from baseline to week 66. Patients receiving inotersen had better/same Norfolk QOL-DN total scores at week 66 compared to baseline more often than did patients receiving placebo $(81.0 \%$ vs. $55.8 \%, p=0.0031)$. Statistically significantly higher percentages of patients with better/ same scores at week 66 than baseline in the inotersen arm than in the placebo arm were also observed for the ADL domain $(81.0 \%$ vs. $51.9 \%, p=0.0005)$, large fiber/physical functioning domain (79.8\% vs. $57.7 \%, p=0.0068)$, and symptoms domain $(84.5 \%$ vs. $65.4 \%, p=0.0121)$. Patients receiving inotersen were more than twice as likely as those receiving placebo to have a meaningfully better Norfolk QOL-DN total score at week 66 than baseline $(25.0 \%$ vs. $9.6 \%, p=0.042$ ). Statistically significantly higher percentages of patients with better scores at week 66 in the inotersen arm than in the placebo arm were also observed for the large

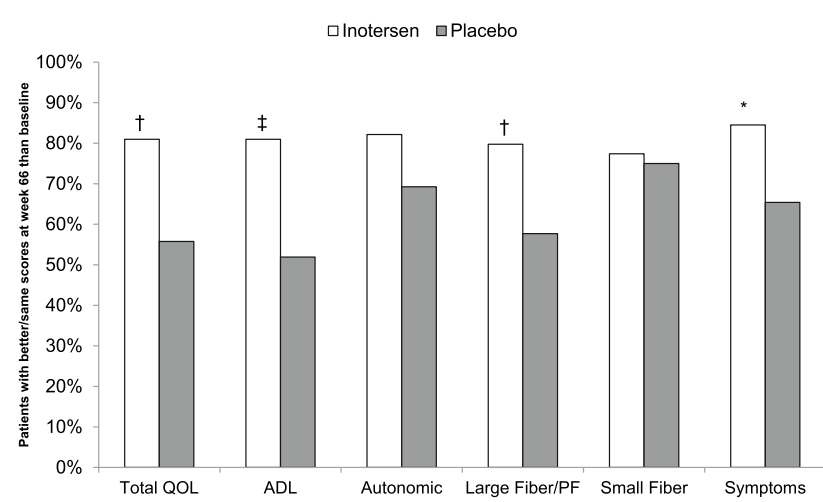

Fig. 3 Responder analysis: percentage of patients with better or same Norfolk QOL-DN Scores at week 66 relative to baseline by treatment arm. $A D L$ activities of daily living, $Q O L$ Quality of Life, $P F$ physical functioning. Better/same scores defined as less than 0.5 of a standard deviation higher at week 66 than at baseline ${ }^{*} p<0.05,{ }^{\dagger} p<0.01$, ${ }^{\ddagger} p<0.001$ fiber/physical functioning domain $(27.4 \%$ vs. $7.7 \%, p=0$. 007).

The percentages of patients whose SF-36v2 domain and summary scores were better or the same at week 66 than baseline are presented in Fig. 4. A significantly larger percentage of patients receiving inotersen than those receiving placebo showed better/same physical and mental summary scores at week $66: 70.1 \%$ vs. $48.1 \%(p=0.0118)$ for PCS and $83.9 \%$ vs. $67.3 \%(p=0.0343)$ for MCS. A statistically significantly larger percentage of inotersen patients than placebo patients showed better/same scores at week 66 on four domains: physical functioning $(83.8 \% \mathrm{vs}$. $50.0 \%, p<0.0001)$, social functioning $(88.5 \%$ vs. $65.4 \%$, $p=0.0018)$, role-emotional ( $81.6 \%$ vs. $63.5 \%, p=0.0256$ ), and mental health $(87.4 \%$ vs. $67.3 \%, p=0.0079)$. Differences in the role-physical $(71.3 \%$ vs. $55.8 \%)$, bodily pain $(80.2 \%$ vs. $65.4 \%)$, and general health $(87.4 \%$ vs. $75.0 \%)$ domains were just outside statistical significance (all $p=0.07$ ). Statistically significantly larger percentages of patients with better scores at week 66 in the inotersen arm than in the placebo arm were observed for the physical functioning domain $(18.4 \%$ vs. $5.8 \%, p=0.042)$ and the role-physical domain (39.1\% vs. $17.3 \%, p=0.008)$.

While the previously described findings were based on Norfolk QOL-DN and SF-36v2 scores at the domain level, additional analyses examined item-level responses for each of the two instruments. At week 66, responses to selected Norfolk QOL-DN items (Table 2) by patients receiving placebo more frequently indicated that they had a moderate or severe problem with a number of symptoms, daily activities, and physical functioning than patients receiving inotersen. For example, patients in the placebo arm indicated pain keeping them awake at night and being bothered by the

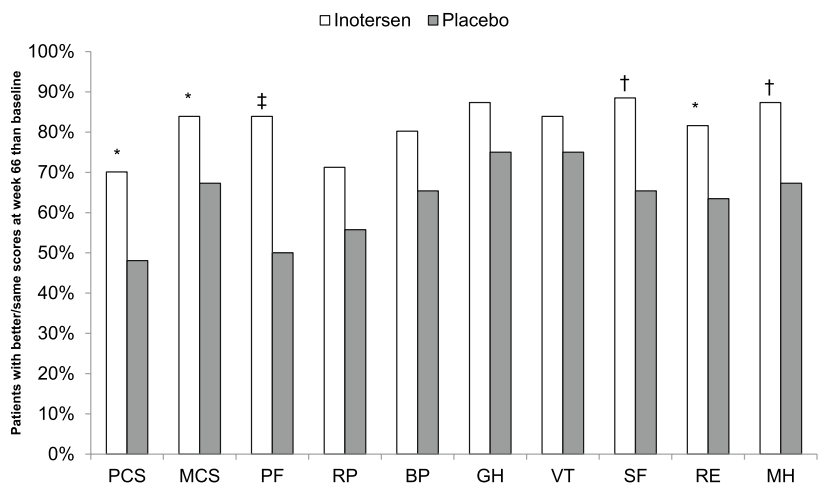

Fig. 4 Responder analysis: percentage of patients with better/same SF-36v2 Scores at week 66 relative to baseline by treatment arm. $B P$ bodily pain, $G H$ general health, $M H$ mental health, $M C S$ mental component summary, $P C S$ physical component summary, $P F$ physical functioning, $R E$ role-emotional, $R P$ role-physical, $S F$ social functioning, $V T$ vitality. Better/same scores defined as greater than the minimally important change (MIC) threshold value lower at week 66 than at baseline ${ }^{*} p<0.05,{ }^{\dagger} p<0.01,{ }^{\ddagger} p<0.001$ 
Table 2 Percentage of patients with Norfolk QOL-DN item responses indicating substantial impairment at week 66, and change in percentage from baseline to week 66 , by treatment arm

\begin{tabular}{|c|c|c|c|c|c|}
\hline \multirow[t]{2}{*}{ Item \# } & \multirow[t]{2}{*}{ Content } & \multicolumn{2}{|l|}{ Week 66} & \multicolumn{2}{|c|}{$\begin{array}{l}\text { Change from baseline to } \\
\text { week } 66\end{array}$} \\
\hline & & Inotersen $(\%)$ & Placebo $(\%)$ & Inotersen $(\%)$ & Placebo (\%) \\
\hline 8 & Pain kept you awake at night & 15.5 & 36.5 & -1.7 & 7.2 \\
\hline 9 & Touch of bed sheets bothered you & 11.9 & 26.9 & -1.6 & 8.0 \\
\hline 10 & Injured yourself without feeling & 14.3 & 19.2 & 1.0 & 5.4 \\
\hline 11 & Symptoms affect usual activity & 35.7 & 50.0 & 6.9 & 20.7 \\
\hline 12 & Difficult movement with fingers & 46.4 & 63.5 & 1.7 & 18.6 \\
\hline 13 & Felt unsteady on your feet & 48.8 & 67.3 & -0.7 & 17.3 \\
\hline 14 & Problem getting out of a chair & 50.0 & 61.5 & 3.3 & 11.5 \\
\hline 15 & Problem walking down stairs & 41.7 & 57.7 & 0.7 & 16.3 \\
\hline 16 & Unable to feel your feet & 45.2 & 40.4 & 10.3 & 0.7 \\
\hline 17 & Unable to tell hot (hands) & 15.5 & 25.0 & 1.1 & 4.3 \\
\hline 18 & Unable to tell hot (feet) & 40.5 & 38.5 & -0.5 & 0.5 \\
\hline 19 & Problem with vomiting & 6.0 & 5.8 & 2.1 & 2.3 \\
\hline 20 & Problem with diarrhea & 20.2 & 30.8 & -1.7 & 15.3 \\
\hline 21 & Problem with fainting/dizziness & 10.7 & 19.2 & -8.3 & 10.6 \\
\hline 22 & Difficulty bathing & 22.6 & 34.6 & -0.2 & 17.4 \\
\hline 23 & Difficulty dressing & 21.4 & 34.6 & 1.4 & 22.5 \\
\hline 24 & Difficulty walking & 40.5 & 59.6 & 3.9 & 28.6 \\
\hline 25 & Difficulty getting on/off toilet & 22.6 & 36.5 & 2.6 & 15.8 \\
\hline 26 & Difficulty using utensils & 19.0 & 30.8 & -1.0 & 10.1 \\
\hline
\end{tabular}

For the selected Norfolk QOL-DN items, substantial impairment was defined as a response of 'moderate problem' or 'severe problem' touch of bedsheets more than twice as often as those receiving inotersen. Descriptively, a noticeably larger percentage of patients (i.e., at least $10 \%$ more) receiving placebo than inotersen reported problems with their symptoms affecting their usual activities; feeling unsteady on their feet; having difficulty moving fingers; having difficulty rising from a chair or descending stairs; having diarrhea; and having difficulty bathing, dressing, walking, getting on/off the toilet, and handling utensils.

Changes from baseline at week 66 reveal increased percentages of patients in the placebo arm exhibiting problems on Norfolk QOL-DN items, while there are mostly small increases, and even slight decreases, in the percentage of patients in the inotersen arm reporting problems (Table 2). Areas where substantial increases in problems in the placebo arm were accompanied by only slight increases, or even decreases in problems in the inotersen arm included difficulty moving fingers, feeling unsteady on their feet, problems descending stairs, problems with diarrhea, problems with dizziness/fainting, and difficulty bathing, dressing, and walking.

Among the selected SF-36v2 items (Table 3), at week 66 patients receiving placebo more frequently (by at least $15 \%$ ) reported being 'limited a lot' in many aspects of physical functioning, including moderate activities; lifting/carrying groceries; climbing several flights of stairs; bending, kneeling, or stooping; and walking several hundred yards than those receiving inotersen. Patients receiving placebo also more frequently reported quite a bit/extreme disruption of physical and emotional health on social activities at week 66 than did patients in the inotersen arm. Changes from baseline at week 66 showed large increases in percentages of patients in the placebo arm experiencing problems, while there were either slight increases or decreases in the percentage of patients in the inotersen arm reporting problems in most aspects of physical functioning and role limitation due to physical health problems, as well as health interference in social functioning (Table 3).

\section{Discussion}

We showed that inotersen treatment preserved or improved QOL over 66 weeks in patients with hATTR amyloidosis with polyneuropathy. Several domains on the Norfolk QOLDN and the SF-36v2 were stable or showed improvements in patients treated with inotersen compared to placebo, for which worsening in mean scores from baseline to week 66 was observed. The effects were more pronounced for physical functioning, role-physical, bodily pain, social functioning, and role-emotional domains. Responder analyses supported these findings. Patients receiving placebo more 
Table 3 Percentage of patients with SF-36v2 item responses indicating substantial impairment at week 66, and change in percentage from baseline to week 66 , by treatment arm

\begin{tabular}{|c|c|c|c|c|c|}
\hline \multirow[t]{2}{*}{ Item \# } & \multirow[t]{2}{*}{ Content } & \multicolumn{2}{|l|}{ Week 66} & \multicolumn{2}{|c|}{$\begin{array}{l}\text { Change from baseline to } \\
\text { week } 66\end{array}$} \\
\hline & & Inotersen $(\%)$ & Placebo (\%) & Inotersen $(\%)$ & Placebo (\%) \\
\hline PF01 & Vigorous activities & 75.9 & 82.7 & -5.3 & 4.7 \\
\hline PF02 & Moderate activities & 42.5 & 59.6 & -3.7 & 22.3 \\
\hline PF03 & Lifting/carrying groceries & 32.6 & 51.9 & -2.3 & 19.7 \\
\hline PF04 & Climbing several flights & 56.3 & 75.0 & -2.2 & 19.1 \\
\hline PF05 & Climbing one flight & 33.3 & 46.2 & 3.1 & 27.5 \\
\hline PF06 & Bending/kneeling/stooping & 39.1 & 63.5 & -1.5 & 27.9 \\
\hline PF07 & Walking more than 1 mile & 59.8 & 65.4 & -1.2 & 14.5 \\
\hline PF08 & Walking several hundred yards & 32.2 & 53.8 & -5.9 & 21.6 \\
\hline PF09 & Walking 100 yards & 24.1 & 32.7 & 6.2 & 19.1 \\
\hline PF10 & Bathing/dressing & 16.1 & 19.2 & 7.6 & 14.1 \\
\hline RP01 & Cut down amount of time working & 32.2 & 55.8 & -6.5 & 25.3 \\
\hline RP02 & Accomplished less & 44.8 & 57.7 & 2.4 & 18.7 \\
\hline RP03 & Limited in kind of work/activity & 43.7 & 59.6 & -2.5 & 12.2 \\
\hline RP04 & Had difficulty performing work/activity & 43.7 & 61.5 & -4.4 & 17.5 \\
\hline SF01 & Health interfered with social activities & 11.5 & 32.7 & -7.6 & 15.7 \\
\hline BP02 & Pain interfered with work/activities & 20.7 & 32.7 & -1.2 & 3.9 \\
\hline
\end{tabular}

For PF items, substantial impairment was defined as a response of 'limited a lot'. For RP items, substantial impairment was defined as a response of 'all of the time' or 'most of the time'. For SF and BP items, substantial impairment was defined as a response of 'extremely' or 'quite a bit'

$B P$ bodily pain, $P F$ physical functioning, $R P$ role-physical, $S F$ social functioning frequently exhibited clinically meaningful worsening in Norfolk QOL-DN total scores, as well as in domains for ADLs, large fiber/physical functioning, and symptoms, while patients receiving inotersen more often showed either clinically meaningful improvements or no change from baseline in the outcomes. A significantly larger percentage of patients receiving inotersen showed improvements on total scores and the large fiber/physical functioning domain than placebo patients. In addition, a significantly larger percentage of patients receiving inotersen were observed to exhibit clinically meaningful improvement for global measures of both SF-36v2 physical (PCS) and mental (MCS) health outcomes, as well as on domains capturing physical functioning, social functioning, role-emotional, and mental health than for those receiving placebo.

Previous analyses of drug treatment for patients with hATTR amyloidosis, including tafamidis [25-29], diflunisal [21], patisiran [30], and inotersen [13], have shown preserved or positive effects on overall QOL. The current analysis, however, is the first to examine the impact of any treatment for hATTR amyloidosis on specific aspects of functioning and activities of daily living.

The current descriptive examination of responses to items on the Norfolk QOL-DN and the SF-36v2 provides important context for interpreting the impact of inotersen on patients' daily activities and functioning. Inotersen was more effective than placebo at preserving or improving outcomes related to sleep; to everyday physical functioning, including walking up and down stairs, carrying groceries, walking, and movement of fingers; having less impairment in social functioning and work-related outcomes; and to having less trouble engaging in everyday activities such as bathing, dressing, and handling eating utensils. Responses to items related to neuropathic pain, such as pain that interferes with sleep or bedsheets rubbing against the skin being a noxious stimulus (allodynia), indicate that inotersen treatment, relative to placebo, significantly improved pain, which is consistent with reduced nerve injury resulting in less neuropathic pain. Patients receiving placebo were more likely to show impairment over time for all of these outcomes, while patients receiving inotersen showed improvements or slight impairments in most of these outcomes from baseline to week 66.

One notable finding from the item-level analysis was a larger difference between inotersen and placebo groups on exhibiting substantial limitations at week 66 on the SF36v2 physical functioning item for walking several hundred yards, yet smaller differences between groups for the items examining walking 100 yards or walking more than a mile. For both of these latter items, slightly more patients in the inotersen group than the placebo group showed substantial difficulties at baseline, which likely led to underestimating the differences at week 66. More revealing is the magnitude of changes from baseline in the percentages of patients 
having substantial difficulty with walking 100 yards (6\% for inotersen vs. $19 \%$ for placebo) or with walking more than a mile ( $-1 \%$ for inotersen (improvement) vs. $15 \%$ for placebo (worsening)).

It should also be noted that there was little change in the impact on QOL of small fiber and autonomic neuropathy throughout the study, even for patients in the placebo arm. The mean increase in the Norfolk QOL-DN small fiber and autonomic neuropathy domains from baseline to week 66 was less than one point in both treatment arms. Further, less than one-third of the sample in the placebo arm showed clinically meaningful worsening in these domains from baseline to week 66. Analysis of change in Norfolk QOL-DN small fiber and autonomic neuropathy scores in the placebo arm of the phase 3 APOLLO study of patients with hATTR amyloidosis showed an increase of 2.8 and 0.8 points, respectively, over 18 months, so also showing minimal change [31]. Thus, it may be that the majority of patients in the NEURO-TTR trial had already reached a critical progression in small fiber and autonomic neuropathy, which are early manifestations of the disease, producing a ceiling effect. At the same time, it should be noted that we did see improvement in autonomic neuropathy for patients receiving inotersen relative to placebo: the proportion of patients in the inotersen arm experiencing autonomic symptoms of fainting/dizziness and diarrhea decreased from baseline to week $66(-8.3 \%$ and $-1.7 \%$, respectively), while the proportion of patients in the placebo arm experiencing these symptoms increased from baseline to week 66 (10.6\% and $15.3 \%$, respectively). Another possibility is that the Norfolk QOL-DN small fiber and autonomic neuropathy domains scores lack the sensitivity needed to detect actual changes in this outcome.

There are limitations of the current study that should be considered. One limitation is the lack of end-stage patients in the NEURO-TTR trial. By the study protocol, only patients in Stage 1 or Stage 2 severity were eligible for enrollment; patients in Stage 3 (not ambulatory) were excluded. It is possible that the impact of inotersen might be different for patients with highly advanced disease than for those in the earlier stages, although it should be noted that many aspects of functioning (e.g., walking, climbing stairs) would not be measurable in non-ambulatory patients. Another limitation was the duration of the treatment period, which was 66 weeks. Patients in either treatment arm who completed the trial were eligible to enroll in an open-label extension (OLE) of the trial in which they would receive $300 \mathrm{mg}$ inotersen once weekly for up to 5 years. Future results from the OLE study will address the long-term impact of inotersen on patients' QOL. The responder-level MIC values for SF-36 domain and summary scores that were used in the responder analyses were established based on a US general population sample, and have not been tested in an hATTR amyloidosis sample, where the magnitude of differences in scores over time corresponding to meaningful change may differ. Also, the designation of item responses characterized as indicating substantial impairment or not substantial impairment was based on arbitrary, subjective judgment; response choices could be dichotomized differently. Finally, we note that all analyses conducted here were post hoc and exploratory; they were performed to better understand the impact of inotersen on more granular components of QOL, since the pre-specified endpoints of the NEURO-TTR study were based on total and summary scores of QOL measures.

In conclusion, patients with hATTR amyloidosis with polyneuropathy treated with inotersen showed better preservation or improvements of QOL at 66 weeks than did patients receiving placebo, particularly with respect to physical functioning, activities of daily living, neuropathic symptoms, role limitations due to physical and emotional functioning, and social functioning.

Acknowledgements We thank the patients who participated in this trial and their families; and Sean O'Connor from Optum for copy-editing and preparing this manuscript for submission.

Author contributions All authors contributed to the study conception and design. Data collection was performed by TC, MWC, CK, SK, MG, GM, LO, HHS, MP, PJBD, THB, IC, MDB, and JLB. Data analyses were performed by AY. The first draft of the manuscript was written by AY and all authors commented on previous versions of the manuscript. All authors read and approved the final manuscript.

Funding This research was funded by Akcea Therapeutics and Ionis Pharmaceuticals, Inc.

\section{Compliance with ethical standards}

Conflicts of interest Coelho, Prothena: consultancy, honoraria; Ionis: consultancy, other: investigator; Alnylam: consultancy, honoraria, other: investigator; Pfizer: consultancy, honoraria, other: investigator. Yarlas, Lovley, Sikora Kessler, and White are employees of Optum, which received payment from Akcea Therapeutics and Ionis Pharmaceuticals Inc. to conduct these analyses and develop this manuscript. Waddington Cruz: Ionis: honoraria; Genzyme/Sanofi: honoraria; Pfizer: honoraria. Pollock is an employee of and owns stock in Akcea Therapeutics. Guthrie was an employee of and owned stock in Akcea Therapeutics. Ackermann, Akcea: consultancy. Hughes is a former employee of and owns stock in Ionis Pharmaceuticals, Inc. Karam, Acceleron: consultancy; Akcea: consultancy; Alexion: consultancy; Alnylam: consultancy; Biogen: consultancy; Cytokinetics: consultancy; CSL Behring: consultancy, Sanofi Genzyme: consultancy. Khella: Akcea: consultancy; Alnylam: consultancy; Pfizer: consultancy. Gertz: Janssen: consultancy; Teva: consultancy; Spectrum: consultancy, honoraria; Alnylam: honoraria; Ionis: honoraria; Amgen: consultancy; Annexon: consultancy; Prothena: honoraria; research to practice: consultancy; Apellis: consultancy; Celgene: consultancy; Abbvie: consultancy; Medscape: consultancy; Physicians Education Resource: consultancy. Merlini: no relationships to disclose. Obici, has received speaking and consulting fees from Akcea, Pfizer and Alnylam. Schmidt, Akcea: consultancy; Alnylam: consultancy; Pfizer: consultancy. Polydefkis, Pfizer: honoraria; Alnylam: honoraria. Dyck, Ionis: consultancy; Alnylam: consultancy. Brannagan, Alnylam: honoraria, other: investigator, Speakers Bureau; Ionis: honoraria, consultancy, speaking, other: investigator. 
Conceição, Ionis: other: investigator; Alnylam: consultancy, honoraria, other: investigator; Pfizer: consultancy, honoraria, other: investigator. Benson, Ionis: other: investigator, research funding. Berk, Ionis: honoraria, other: investigator; Alnylam: honoraria, other: investigator; Pfizer: other: investigator.

Ethical approval The trial protocol was approved by institutional review boards or local ethics committees. The trial was conducted in accordance with Good Clinical Practice guidelines of the International Conference on Harmonisation and the principles of the Declaration of Helsinki. All the patients provided written informed consent to participate in the trial.

Open Access This article is licensed under a Creative Commons Attribution 4.0 International License, which permits use, sharing, adaptation, distribution and reproduction in any medium or format, as long as you give appropriate credit to the original author(s) and the source, provide a link to the Creative Commons licence, and indicate if changes were made. The images or other third party material in this article are included in the article's Creative Commons licence, unless indicated otherwise in a credit line to the material. If material is not included in the article's Creative Commons licence and your intended use is not permitted by statutory regulation or exceeds the permitted use, you will need to obtain permission directly from the copyright holder. To view a copy of this licence, visit http://creativecommons.org/licenses/by/4.0/.

\section{References}

1. Hawkins PN, Ando Y, Dispenzeri A et al (2015) Evolving landscape in the management of transthyretin amyloidosis. Ann Med 47(8):625-638. https://doi.org/10.3109/07853890.2015.1068949

2. Sekijima Y (2015) Transthyretin (ATTR) amyloidosis: clinical spectrum, molecular pathogenesis and disease-modifying treatments. J Neurol Neurosurg Psychiatry 86(9):1036-1043. https:// doi.org/10.1136/jnnp-2014-308724

3. Gertz MA (2017) Hereditary ATTR amyloidosis: burden of illness and diagnostic challenges. Am J Manag Care 23(7 Suppl):S107-S112

4. Conceição I, González-Duarte A, Obici L et al (2016) "Red-flag" symptom clusters in transthyretin familial amyloid polyneuropathy. J Peripher Nerv Syst 21(1):5-9. https://doi.org/10.1111/ jns. 12153

5. Planté-Bordeneuve V, Said G (2011) Familial amyloid polyneuropathy. Lancet Neurol 10(12):1086-1097. https://doi. org/10.1016/S1474-4422(11)70246-0

6. Rapezzi C, Quarta CC, Riva L et al (2010) Transthyretin-related amyloidoses and the heart: a clinical overview. Nat Rev Cardiol 7(7):398-408. https://doi.org/10.1038/nrcardio.2010.67

7. Coelho T, Maurer MS, Suhr OB (2013) THAOS-The Transthyretin Amyloidosis Outcomes Survey: initial report on clinical manifestations in patients with hereditary and wild-type transthyretin amyloidosis. Curr Med Res Opin 29(1):63-76. https:// doi.org/10.1185/03007995.2012.754348

8. Drent G, Graveland CW, Hazenberg BPC et al (2009) Quality of life in patients with familial amyloidotic polyneuropathy longterm after liver transplantation. Amyloid 16(3):133-141. https:// doi.org/10.1080/13506120903090726

9. Stewart M, Shaffer S, Murphy B et al (2018) Characterizing the high disease burden of transthyretin amyloidosis for patients and caregivers. Neurol Ther. https://doi.org/10.1007/s4012 $0-018-0106-z$
10. Lovley A, Guthrie SD, Sikora Kessler A et al (2018) The burden of heriditary transthyretin amyloidosis on health-related quality of life. Presented at ISPOR Annual Meeting, Baltimore, MD, USA, 19-23 May, 2018

11. Yarlas A, Gertz M, Dasgupta NR et al (2018) Burden of hereditary transthyretin amyloidosis on quality of life. Muscle Nerve 60(2):169-175. https://doi.org/10.1002/mus.2651

12. Coelho T, Vinik A, Vinik EJ et al (2017) Clinical measures in transthyretin familial amyloid polyneuropathy. Muscle Nerve 55(3):323-332. https://doi.org/10.1002/mus.25257

13. Benson MD, Waddington-Cruz M, Berk JL et al (2018) Inotersen treatment for patients with hereditary transthyretin amyloidosis. $\mathrm{N}$ Engl J Med 379(1):22-31. https://doi.org/10.1056/NEJMoa1716 793

14. Coutinho P, Martins da Silva A, Lopes Lima J et al (1980) Forty years of experience with type 1 amyloid neuropathy. Review of 483 cases. In: Glenner G, Costa P, Freitas A (eds) Amyloid and amyloidosis. Execerpta Medica, Amsterdam, pp 88-98

15. Dyck PJ, Kratz KM, Lehman KA et al (1991) The Rochester Diabetic Neuropathy Study: design, criteria for types of neuropathy, selection bias, and reproducibility of neuropathic tests. Neurology 41(6):799-807

16. Dyck PJ, Kincaid JC, Dyck P et al (2017) Assessing mNIS+7Ionis and international neurologists' proficiency in a familial amyloidotic polyneuropathy trial. Muscle Nerve 56(5):901-911. https:// doi.org/10.1002/mus. 25563

17. Suanprasert N, Berk JL, Benson MD et al (2014) Retrospective study of a TTR FAP cohort to modify NIS +7 for therapeutic trials. J Neurol Sci 344(1-2):121-128. https://doi.org/10.1016/j. jns.2014.06.041

18. Vinik EJ, Hayes RP, Oglesby A et al (2005) The development and validation of the Norfolk QOL-DN, a new measure of patients' perception of the effects of diabetes and diabetic neuropathy. Diabetes Technol Ther 7(3):497-508. https://doi.org/10.1089/ dia.2005.7.497

19. Vinik EJ, Vinik AI, Paulson JF et al (2014) Norfolk QOL-DN: validation of a patient reported outcome measure in transthyretin familial amyloid polyneuropathy. J Peripher Nerv Syst 19(2):104 114. https://doi.org/10.1111/jns5.12059

20. Maruish ME (2011) User's manual for the SF-36v2 health survey, 3rd edn. QualityMetric Incorporated, Lincoln

21. Berk JL, Suhr OB, Obici L et al (2013) Repurposing diflunisal for familial amyloid polyneuropathy: a randomized clinical trial. JAMA 310(24):2658-2667. https://doi.org/10.1001/ jama.2013.283815

22. Jaeschke R, Singer J, Guyatt GH (1989) Measurement of health status. Ascertaining the minimal clinically important difference. Control Clin Trials 10(4):407-415

23. Norman GR, Sloan JA, Wyrwich KW (2003) Interpretation of changes in health-related quality of life: the remarkable universality of half a standard deviation. Med Care 41(5):582-592. https:// doi.org/10.1097/01.MLR.0000062554.74615.4C

24. Norman GR, Sloan JA, Wyrwich KW (2004) The truly remarkable universality of half a standard deviation: confirmation through another look. Expert Rev Pharmacoecon Outcomes Res 4(5):581585. https://doi.org/10.1586/14737167.4.5.581

25. Ando Y, Sekijima Y, Obayashi K et al (2016) Effects of tafamidis treatment on transthyretin (TTR) stabilization, efficacy, and safety in Japanese patients with familial amyloid polyneuropathy (TTR-FAP) with Val30Met and non-Val30Met: A phase III, openlabel study. J Neurol Sci 362:266-271. https://doi.org/10.1016/j. jns.2016.01.046

26. Coelho T, Maia LF, da Silva AM et al (2013) Long-term effects of tafamidis for the treatment of transthyretin familial amyloid polyneuropathy. J Neurol 260(11):2802-2814. https://doi.org/10.1007/ s00415-013-7051-7 
27. Mundayat R, Stewart M, Alvir J et al (2018) Positive effectiveness of tafamidis in delaying disease progression in transthyretin familial amyloid polyneuropathy up to 2 years: an analysis from the Transthyretin Amyloidosis Outcomes Survey (THAOS). Neurol Ther 7(1):87-101. https://doi.org/10.1007/s40120-018-0097-9

28. Barroso FA, Judge DP, Ebede B et al (2017) Long-term safety and efficacy of tafamidis for the treatment of hereditary transthyretin amyloid polyneuropathy: results up to 6 years. Amyloid 24(3):194-204. https://doi.org/10.1080/13506129.2017.1357545

29. Merlini G, Planté-Bordeneuve V, Judge DP et al (2013) Effects of tafamidis on transthyretin stabilization and clinical outcomes in patients with non-Val30Met transthyretin amyloidosis. J Cardiovasc Transl Res 6(6):1011-1020. https://doi.org/10.1007/s1226 5-013-9512-x

30. Adams D, Gonzalez-Duarte A, O'Riordan WD et al (2018) Patisiran, an RNAi therapeutic, for hereditary transthyretin amyloidosis. N Engl J Med 379(1):11-21. https://doi.org/10.1056/NEJMo a1716153

31. Dyck PJ, Adams D, Coelho T et al (2018) Neuropathy progression in patients with hATTR amyloidosis: analysis of the APOLLO placebo arm. Presented at Peripheral Nervous Society Annual Meeting, Baltimore, MD, USA, 22-25 July, 2018

\section{Affiliations}

Teresa Coelho ${ }^{1} \cdot$ Aaron Yarlas $^{2}(1) \cdot$ Marcia Waddington-Cruz ${ }^{3} \cdot$ Michelle K. White $^{2} \cdot$ Asia Sikora Kessler $^{2}$. Andrew Lovley ${ }^{2}$. Michael Pollock ${ }^{4}$. Spencer Guthrie ${ }^{5}$. Elizabeth J. Ackermann ${ }^{6}$ - Steven G. Hughes ${ }^{7}$. Chafic Karam ${ }^{8}$. Sami Khella ${ }^{9}$. Morie Gertz ${ }^{10}$. Giampaolo Merlini ${ }^{11}$ - Laura Obici ${ }^{11} \cdot$ Hartmut H. Schmidt ${ }^{12} \cdot$ Michael Polydefkis $^{13}$. P. James B. Dyck ${ }^{14}$. Thomas H. Brannagan III ${ }^{15}$. Isabel Conceição ${ }^{16}$. Merrill D. Benson ${ }^{17}$. John L. Berk ${ }^{18}$

1 Hospital Santo António, Centro Hospitalar Universitário do Porto, Porto, Portugal

2 Optum, 1301 Atwood Avenue, Johnston, RI 02919, USA

3 Hospital Universitário Clementino Fraga Filho, Universidade Federal do Rio de Janeiro, Rio de Janeiro, Brazil

4 Akcea Therapeutics, Boston, MA, USA

5 Aurora Bio, San Francisco, CA, USA

6 Otonomy, Inc., San Diego, CA, USA

7 Organovo Holdings, Inc., San Diego, CA, USA

8 Department of Neurology, Oregon Health and Science University, Portland, OR, USA

9 University of Pennsylvania, Philadelphia, PA, USA

10 Division of Hematology, Mayo Clinic, Rochester, MN, USA
11 Amyloidosis Research and Treatment Center, Fondazione IRCCS Policlinico San Matteo, University of Pavia, Pavia, Italy

12 University of Münster, Münster, Germany

13 Johns Hopkins University School of Medicine, Baltimore, MD, USA

14 Department of Neurology, Mayo Clinic, Rochester, MN, USA

15 Columbia University, New York, NY, USA

16 Faculdade de Medicina, Hospital de Santa Maria, CHULN, Universidade de Lisboa, Lisboa, Portugal

17 Indiana University School of Medicine, Indianapolis, IN, USA

18 Boston University School of Medicine, Boston, MA, USA 